\title{
Sinterização e caracterização de segunda fase em sistemas $\mathrm{SnO}_{2}-\mathrm{ZnO}$
}

\section{(Sintering and second phase assignments in the $\mathrm{SnO}_{2}-\mathrm{ZnO}$ system)}

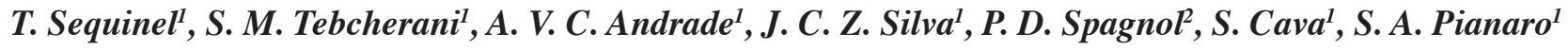 \\ ${ }^{I}$ CIPP - LIMAC - DEQUIM, Universidade Estadual de Ponta Grossa, Ponta Grossa, PR \\ ${ }^{2}$ LIEC - Instituto de Química - UNESP, Araraquara, SP \\ sergiomt@uepg.br
}

\begin{abstract}
Resumo
A adição de $\mathrm{ZnO}$ em sistemas de $\mathrm{SnO}_{2}$ faz com que estas cerâmicas atinjam elevada densificação quando adicionadas em baixas concentrações. Neste trabalho estudou-se o comportamento da adição do óxido de zinco desde 0,5 até 2,0 mol\%. Desta forma, podese verificar que na sinterização em dilatômetro até $1365^{\circ} \mathrm{C}$ a adição de até $1,5 \mathrm{~mol} \%$ favorece a densificação da cerâmica e, quando a adição do $\mathrm{ZnO}$ é superior a essa concentração o efeito densificante passa a ter um caráter reduzido em $14 \%$ da retração linear. Tal comportamento justificou-se pela formação de uma segunda fase de estanato de zinco em quantidade próxima a $2 \mathrm{~mol} \%$ de $\mathrm{ZnO}$ quando analisado por difração de raios X.
\end{abstract}

Palavras-chave: sinterização, $\mathrm{SnO}_{2}$, difração de raios $\mathrm{X}$.

\begin{abstract}
Small $\mathrm{ZnO}$ additions in $\mathrm{SnO}_{2}$ systems result in ceramics with high densification. The aim of this work is to study the behavior of zinc addition ranging from 0.5 to $2 \mathrm{mol \%} \mathrm{SnO}_{2}$ obtained by the Pechini method. The addition of $1.5 \mathrm{~mol} \% \mathrm{ZnO}$ assisted in the densification of the ceramic. However, further additions inhibited the process reducing the linear shrinkage by $14 \%$. This behavior is justified by the formation of a second phase of zinc stannate in quantities close to $2 \mathrm{~mol} \%$ as estimated by X-ray diffraction determining the lattice parameters of the $\mathrm{SnO}_{2}-\mathrm{ZnO}$ system.
\end{abstract}

Keywords: sintering, $\mathrm{SnO}_{2}$, X-ray diffraction.

\section{INTRODUÇÃO}

Tanto o dióxido de estanho como o óxido de zinco são os dois óxidos mais utilizados como sensores de gases [1]. Devido apresentar caráter covalente, $\mathrm{o} \mathrm{SnO}_{2}$ é inerte à maioria das corrosões químicas habilitando-o a aplicação em fornos de vidros. Associando esta característica a propriedade adquirida em presença de alguns aditivos como nióbio e antimônio, torna-o excelente material cerâmico para aplicação como varistor [2,3]. As propriedades de cerâmicas varistoras também são conferidas ao $\mathrm{ZnO}$ que são comercializadas atualmente.

Pelo baixo custo que é encontrado, o dióxido de estanho faz com que as investigações quanto suas aplicabilidades sejam intensificadas. Das formas de obtenção do dióxido de estanho, a rota química dos precursores poliméricos, também conhecido como método Pechini [4], é bastante eficaz quanto a incorporação de aditivos na rede do $\mathrm{SnO}_{2}$, apresentando uma distribuição homogênea mesmo que em quantidades ínfimas [5].

O processo de sinterização é o resultado da redução da área de superfície pela formação do contorno de grão, crescimento dos pescoços entre as partículas seguidas ou não de densificação.
A sinterização é estudada em três estágios distintos [6]: inicial, intermediário e final. O estágio inicial é definido como a parte do processo onde ocorre o arredondamento das partículas, a formação de pescoços ou de contornos entre as mesmas, o desenvolvimento dos pescoços com pouco crescimento de grão e uma significativa redução tanto na área superficial livre e da porosidade. Este estágio avança até o ponto onde os pescoços interferem uns com os outros.

No estágio intermediário ocorre acentuado crescimento de grão e fechamento de poros acompanhado de densificação devido à ação das respectivas energias de tensões superficiais.

O estágio final é caracterizado pela eliminação de poros residuais com pouca ou nenhuma densificação. Quando ela ocorre, observa-se o crescimento de grãos.

Sabe-se que, o óxido de estanho quando puro não densifica durante a sinterização [7]. Tem sido encontrado que a adição de cátions como: $\mathrm{Mn}, \mathrm{Fe}, \mathrm{Cu}, \mathrm{Co}, \mathrm{Zn}$, dentre outros, que levam a um aumento da superfície específica do $\mathrm{SnO}_{2}$, criando defeitos pontuais ativando a densificação da cerâmica [8]. Acredita-se que a incorporação desses aditivos produz defeitos na superfície do $\mathrm{SnO}_{2}$ correspondentes a espécies de estanho ou oxigênio que levam a uma maior mobilidade na rede resultando em densificação. Entretanto 
se torna mais possível quando os defeitos estão relacionados à deficiência de oxigênio na superfície dos grãos $[9,10]$ de $\mathrm{SnO}_{2}$. Azad [11] estabeleceu uma correlação entre o processamento e a microestrutura de cerâmicas obtidas a partir da mistura de estanho metálico com nitratos de cálcio, estrôncio e bário calcinado na proporção 1:1 propondo uma fórmula geral resultante como sendo $\mathrm{MSnO}_{3}$, onde $\mathrm{M}$ representa o cátion alcalino-terroso. Para esses compostos observou uma transição gradual nas condições de sinterização, onde a diferença do raio iônico dos cátions afeta a cinética de sinterização via difusão no estado sólido e é capaz de formar com o estanho um composto secundário de grande estabilidade química [11].

A adição do óxido de zinco ao dióxido de estanho pode superar a $98 \%$ da densidade teórica, prevista para este sistema.

Desta forma, procurou-se investigar neste trabalho o processo de sinterização do $\mathrm{SnO}_{2}$ via método Pechini, dopado com concentrações que variaram de 0,5 até $2,0 \% \mathrm{em}$ mol de $\mathrm{ZnO}$. As sinterizações foram realizadas em dilatômetro desde a temperatura ambiente até $1350{ }^{\circ} \mathrm{C}$ com taxa constante de aquecimento em temperatura ambiente.

\section{MATERIAIS E MÉTODOS}

Através da mistura de cloreto de estanho, ácido cítrico e etilenoglicol obteve-se o citrato de estanho que é insolúvel em água. $\mathrm{O}$ citrato de estanho foi submetido a sucessivas lavagens com água para eliminação de todo o cloreto. A análise da presença de cloreto foi feita com adição de nitrato de prata sobre o filtrado indicando teste negativo para a presença de precipitado branco de cloreto de prata. Ao citrato de estanho foram acrescidas quantidades estequiométricas de ácido cítrico dissolvido em etilenoglicol até obtenção de solução Pechini de citrato de estanho.

Desta solução realizou-se análise gravimétrica para adição de nitrato de zinco em concentrações de $0,5,1,0$, 1,5 e 2,0 mol\%. Após a adição dos densificantes a solução foi aquecida para a formação do poliéster de estanho. Em seguida o material foi calcinado na temperatura de $700{ }^{\circ} \mathrm{C}$ durante 180 minutos para formação do dióxido de estanho dopado. A área de superfície específica do pó foi determinada a partir do método B.E.T. (Quantachrome, Nova 1200).

O óxido foi compactado cilindricamente até a pressão de $210 \mathrm{MPa}$. Os compactos foram medidos geometricamente para determinação da densidade à verde.

As sinterizações dos compactos foram realizadas em dilatômetro horizontal (Netzsch, 402 EP) com taxa de aquecimento constante de $5,0 \% \mathrm{~min}$. desde a temperatura ambiente até $1365^{\circ} \mathrm{C}$.

O óxido dopado também foi caracterizado por difração de raios X. Os dados de difração de raios X foram obtidos num difratômetro Shimadzu XRD-6000, utilizando geometria Bragg-Brentano, de $22^{\circ}$ to $120^{\circ}$ com passo de $0,02^{\circ}$, tempo de $4 \mathrm{~s}$ por passo, fenda de divergência igual a $0,5^{\circ}$ e fenda

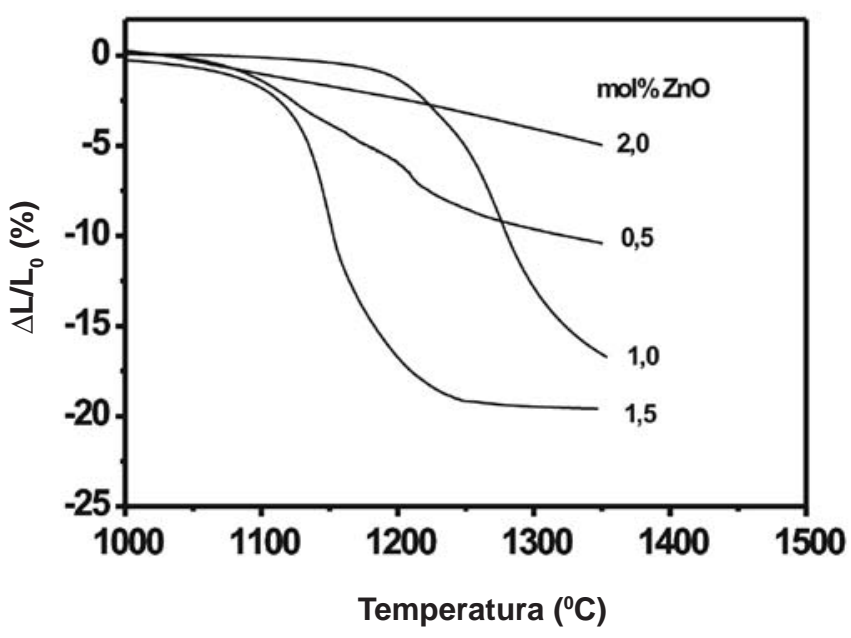

Figura 1: Curvas de retração linear do $\mathrm{SnO}_{2}$ dopado com 0,5 até 2,0 $\mathrm{mol} \%$ de $\mathrm{ZnO}$ com taxa de aquecimento constante $\left(5,0^{\circ} \mathrm{C} / \mathrm{min}\right)$ até $1365^{\circ} \mathrm{C}$.

[Figure 1: Linear shrinkage curves of $\mathrm{SnO}_{2}$ ceramics doped with 0.5 up to $2.0 \mathrm{~mol} \%$ of $\mathrm{ZnO}$ sintered with constant heating rate $\left(5.0^{\circ} \mathrm{C} / \mathrm{min}\right.$ ) up to $1365^{\circ} \mathrm{C}$.]

de recepção igual a $0,15 \mathrm{~mm}$. Foi utilizado tubo de cobre operando a $40 \mathrm{kV}$ e $30 \mathrm{~mA}$, com radiação monocromatizada por cristal de grafite.

O difratograma obtido permitiu a identificação de duas fases: $\mathrm{SnO}_{2}$ com simetria tetragonal e grupo espacial $\mathrm{P} 4_{2} / \mathrm{m} \mathrm{n} \mathrm{m}$, e $\mathrm{Zn}_{2} \mathrm{SnO}_{4}$ com simetria cúbica e grupo espacial $\mathrm{F} \mathrm{d} \overline{3} \mathrm{~m}$. Os dados estruturais dessas duas fases foram obtidos no banco de dados de estruturas ICSD [12].

Com o objetivo de confirmar a presença das fases identificadas e de quantificá-las, foi realizado o refinamento de estrutura pelo método de Rietveld [13, 14] utilizando o programa Rietan-2000 [15], executado sob sistema operacional Windows $\mathrm{ME}^{\oplus}$. Para o ajuste do perfil foi utilizada a função split-pseudo-Voigt.

\section{RESULTADOS E DISCUSSÃO}

Após a preparação do dióxido de estanho pelo método Pechini dopado com 0,5 até 2,0 mol\% de óxido de zinco, chegou-se a área de superfície específica, do óxido na forma de pó, determinado pelo método B.E.T. em $28 \mathrm{~m}^{2} / \mathrm{g}$. A densidade do material após compactação foi da ordem de $58 \%$ da densidade à verde.

A sinterização do dióxido de estanho dopado com 0,5 , $1,0,1,5$ e 2,0 mol\% de $\mathrm{ZnO}$, realizado desde a temperatura ambiente até $1365{ }^{\circ} \mathrm{C}$ usando de taxa de aquecimento constante de $5,0^{\circ} \mathrm{C} / \mathrm{min}$ está representado na Fig. 1.

A Fig. 1 representa a variação da concentração de dopante na mistura de óxido. Pode-se perceber que na medida em que se aumenta a concentração de $\mathrm{ZnO}$ o processo de densificação é aumentado. Esta correlação é 


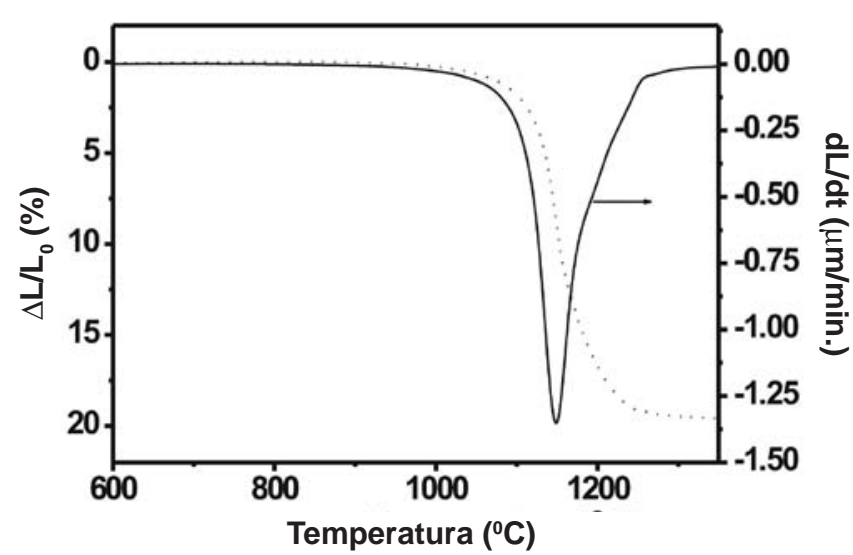

Figura 2: Curva de retração linear e respectiva derivada para sistemas de $\mathrm{SnO}_{2}$ contendo $1,5 \mathrm{~mol} \%$ de $\mathrm{ZnO}$.

[Figure 2: Linear shrinkage curve and respective derivative for $\mathrm{SnO}_{2}$ systems containing $1.5 \mathrm{~mol} \% \mathrm{ZnO}$.]

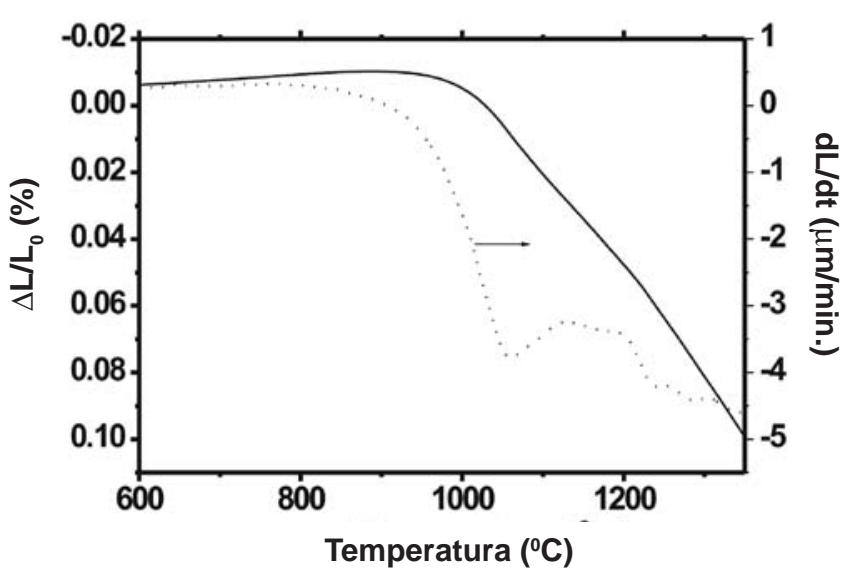

Figura 3: Curva de retração linear e respectiva derivada para sistemas de $\mathrm{SnO}_{2}$ contendo 2,0 mol\% de $\mathrm{ZnO}$.

[Figure 3: Linear shrinkage curve and respective derivative for $\mathrm{SnO}_{2}$ systems containing $2.0 \mathrm{~mol} \% \mathrm{ZnO}$.]

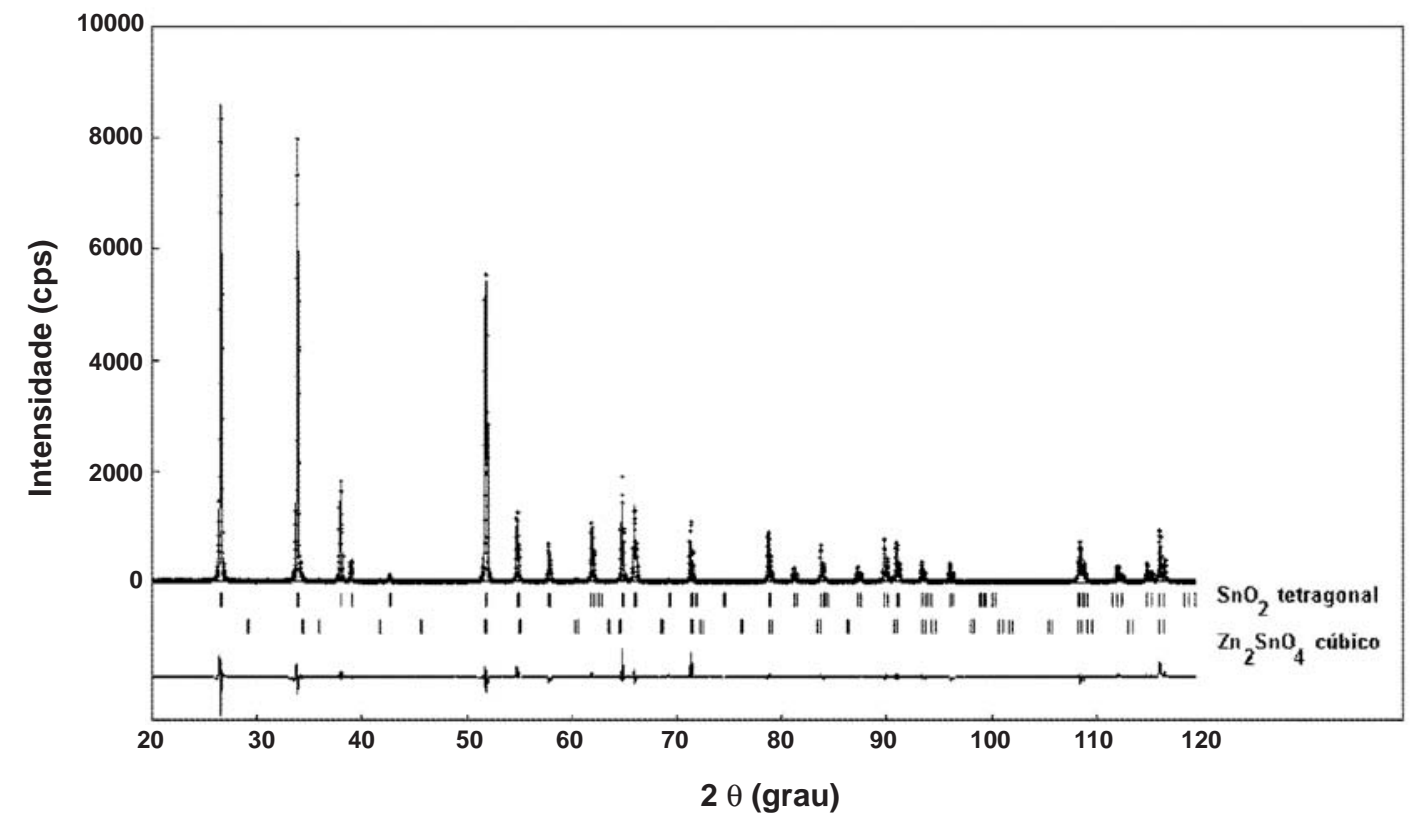

Figura 4: Gráfico de Rietveld após o refinamento realizado com amostra de $\mathrm{SnO}_{2}$ dopada com 2,0 mol\% de $\mathrm{ZnO}$ e tratada termicamente a $1160{ }^{\circ} \mathrm{C}$. [Figure 4: Rietveld plot after refining accomplished with the $\mathrm{SnO}_{2}$ sample doped with $2.0 \mathrm{~mol} \% \mathrm{ZnO}$ and thermally treated at $1160{ }^{\circ} \mathrm{C}$.]

válida até a concentração de $1,5 \mathrm{~mol} \%$ de $\mathrm{ZnO}$ pois, em compactos sinterizados com $2,0 \mathrm{~mol} \%$ de $\mathrm{ZnO}$ o efeito de densificação fica subtraído. Assim, a análise da Fig. 1 leva a investigação para cada curva de sinterização em separado. Desta forma, de acordo com a Fig. 2 percebe-se que, para sistemas que contém 1,5 mol\% de $\mathrm{ZnO}$, a taxa da retração indica o ponto de máxima taxa de retração linear próximo a $1130{ }^{\circ} \mathrm{C}$ e se completa indicando que não existe perda de massa no processo de sinterização.

Já na curva de sinterização para sistemas com 2,0 mol\% de $\mathrm{ZnO}$, observa-se que o término do ensaio atinge somente o estágio inicial de sinterização alterando também a máxima taxa de retração linear, conforme a Fig. 3.

Em discordância com os resultados, viu-se a necessidade de investigação para os sistemas dopados com 2,0 $\mathrm{mol} \%$ de $\mathrm{ZnO}$ usando-se do refinamento de Rietveld para difratogramas de raios $\mathrm{X}$ em amostras na forma de pó quando tratadas termicamente até $1160{ }^{\circ} \mathrm{C}$.

Na Tabela I estão os resultados obtidos após o refinamento de Rietveld da estrutura da fase majoritária $\mathrm{SnO}_{2}$. Os números entre parênteses indicam o desvio padrão de cada medida. Os resultados obtidos para os índices R's indicam a boa qualidade do refinamento.

A análise quantitativa de fases resultou em $98 \%$ em massa 
Tabela I - Resultados obtidos após o refinamento de Rietveld realizado com a amostra de $\mathrm{SnO}_{2}$ dopada com 2,0 mol $\%$ de zinco e tratada termicamente a $1160{ }^{\circ} \mathrm{C}$.

[Table I - Results obtained after Rietveld refining carried out with the $\mathrm{SnO}_{2}$ sample doped with zinc $2.0 \mathrm{~mol} \%$ and thermally treated at $1160^{\circ} \mathrm{C}$.]

\begin{tabular}{ccccccc}
\hline \multicolumn{7}{c}{$\mathrm{a}=4,73709(4) \AA ; \mathrm{c}=3,18616(3) \AA ; \alpha=90^{\circ} ; \mathrm{V}=71.497(1) \AA^{3}$} \\
\hline Átomos & Wyckoff & $\mathbf{X}$ & $\mathbf{Y}$ & $\mathbf{z}$ & sof & $\mathbf{B}\left(\AA^{2}\right)$ \\
Sn & $2 \mathrm{a}$ & 0,0 & 0,0 & 0,0 & 1,0 & 0,228 \\
$\mathrm{O}$ & $4 \mathrm{f}$ & $0,3133(7)$ & $0,3133(7)$ & 0,0 & 1,0 & 0,585 \\
& $\mathrm{Rp}=10,02 \% ; \operatorname{Rwp}=14,92 \% ; \mathrm{Re}=10,40 \% ; \mathrm{S}=1,43 ; \mathrm{R}_{\mathrm{B}}=1,96 ;$ razão c/a = 0,6726 \\
\hline
\end{tabular}

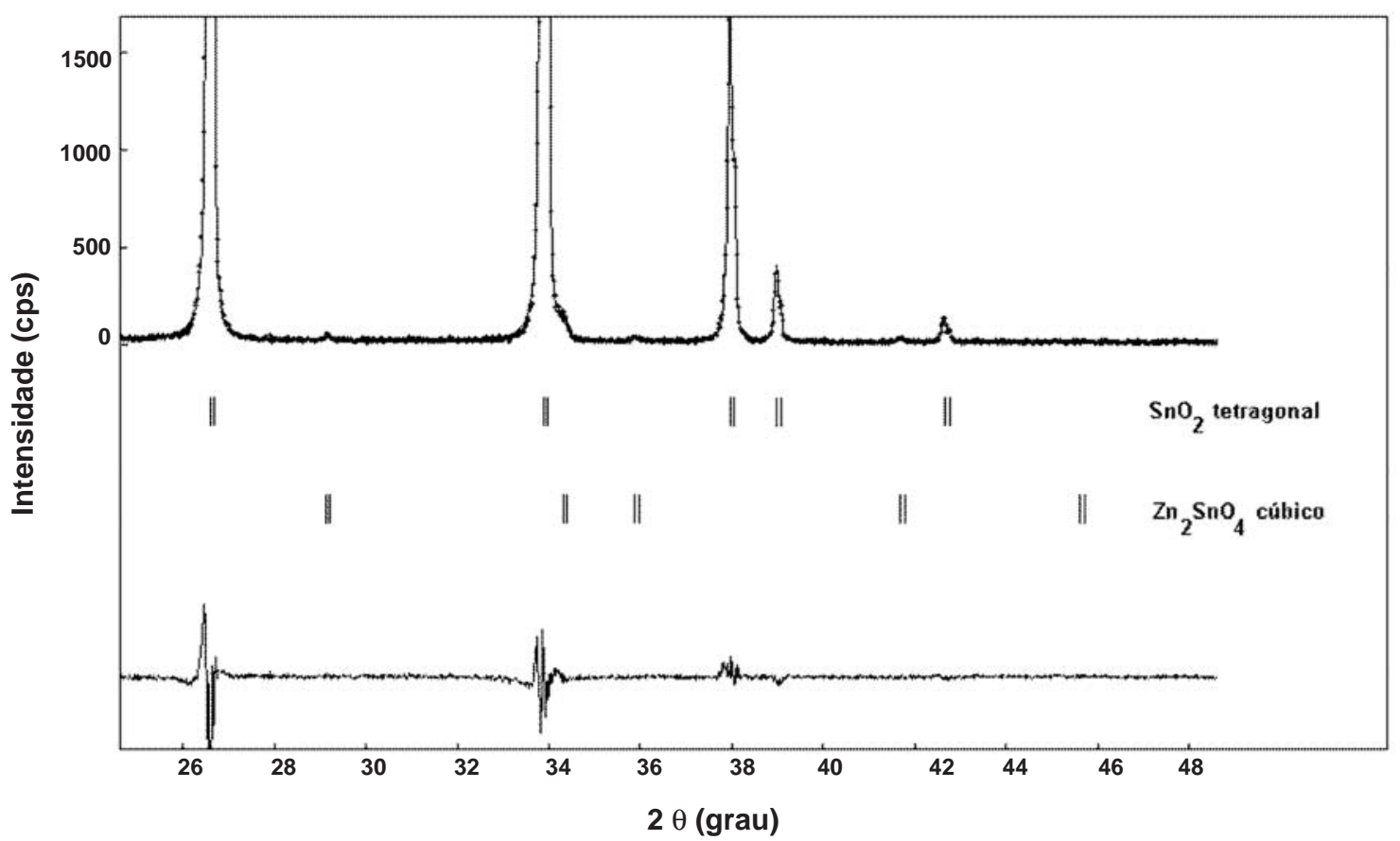

Figura 5: Destaque do gráfico de Rietveld indicando a presença da segunda fase, $\mathrm{Zn}_{2} \mathrm{SnO}_{4}$ cúbica, além da fase $\mathrm{SnO}_{2}$ tetragonal.

[Figure 5: Highlight of Rietveld graph indicating the presence of cubic $\mathrm{Zn}_{2} \mathrm{SnO}_{4}$ as second phase, in addition to tetragonal $\mathrm{SnO}_{2}$. ]

da fase $\mathrm{SnO}_{2}$ tetragonal e $2 \%$ da fase $\mathrm{Zn}_{2} \mathrm{SnO}_{4}$ cúbica.

$\mathrm{O}$ gráfico de Rietveld após o término do refinamento está na Fig. 4. Observa-se que alguns picos não apresentam um bom ajuste, isto se deve, provavelmente, a flutuações na estrutura cristalina do $\mathrm{SnO}_{2}$ tetragonal.

Na Fig. 5 está um destaque do gráfico de Rietveld na região entre $25^{\circ}$ e $48^{\circ}$. Neste destaque observa-se nitidamente a presença da segunda fase $\mathrm{Zn}_{2} \mathrm{SnO}_{4}$ cúbica. As barras verticais duplas indicam as reflexões de Bragg devido às contribuições $\mathrm{K} \alpha_{1}$ e $\mathrm{K} \alpha_{2}$ da radiação característica de cobre.

\section{CONCLUSÃO}

As curvas de sinterização obtidas por dilatometria levantam indícios de alteração na composição de sistema de dióxido de estanho dopado com 2,0 mol\% de $\mathrm{ZnO}$. As características das curvas de sinterização consistem basicamente na significativa redução da densificação que levam a acreditar na formação de substância inibidora do transporte de massa via contorno de grão para o dióxido de estanho. Análises realizadas por Rietveld são capazes determinar a formação de segunda fase de estanato de zinco para o sistema quando o óxido na forma de pó é calcinado acima da máxima taxa da retração linear do sistema óxido de melhor retração linear.

\section{AGRADECIMENTOS}

Os autores deste trabalho agradecem ao Instituto de Química de Araraquara (UNESP), Paraná Tecnologia e CNPq. 


\section{REFERÊNCIAS}

[1] N. Yamazoe, T. Fughigami, N. Kishikama, T. Seiyama, T. Surface Sci. 86 (1979) 335-340.

[2] P. H. Duvigneaud, D. Reighard, In: P. Vicenzini, Ed. Science of sintering, Ceramurgia Srl, Faenza 12 (1980) 287292.

[3] P. R. Bueno, M. M. Oliveira, M. R. Cassia-Santos, E. Longo, S. M. Tebcherani, J. A. Varela, Cerâmica 46, 299 (2000) 124-130.

[4] M. M. Besso, US Patent 3123120 (19/10/1965).

[5] D. Gouvêa, Tese de Doutorado em Físico-Química, Universidade Federal de S. Carlos (1995) 142 p.

[6] L. M. Levinson, H. R. Philipp, Am. Ceram . Soc. Bull. 65, 4 (1986) 639-646.
[7] Y. Chiang, D. Birnie, W. D. Kingery, John Wiley \&Sons Inc., New York (1997) 522.

[8] C. Xu, J. Tamaki, M. Miura, N. Yamazoe, J. Mater. Sci. 27, 4 (1992) 963-971.

[9] J. Nowotny, L. C. Defour, Mater. Sci. Monographs 47 (1988) 194.

[10] E. W. Thornton, P. G. Macavoy, J. Appl. Phys. 32, 12 (1961) 2504.

[11] A. M. Azad, L. L. W. Shyan, T. Y. Pang, C. H. Nee, Ceram. Int. 26, 7 (2000) 685-692.

[12] ICSD: Inorganic Crystal Structure Database, USA, NIST (2002) cd-rom.

[13] H. M. Rietveld, Acta Cryst. 22 (1967) 151-152.

[14] H. M. Rietveld, J. Appl. Cryst. 2 (1969) 65-71.

[15] F. Izumi, J. Rigaku 1 (2000) 34-45. 